\title{
Bypassing dynamical systems: a simple way to get the box-counting dimension of the graph of the Weierstrass function
}

\author{
Claire David
}

Abstract. In the following, bypassing dynamical systems tools, we propose a simple means of computing the box dimension of the graph of the classical Weierstrass function defined, for any real number $x$, by

$$
\mathcal{W}(x)=\sum_{n=0}^{+\infty} \lambda^{n} \cos \left(2 \pi N_{b}^{n} x\right),
$$

where $\lambda$ and $N_{b}$ are two real numbers such that $0<\lambda<1, N_{b} \in \mathbb{N}$ and $\lambda N_{b}>1$, using a sequence a graphs that approximate the studied one.

Анотація. В роботі пропонується простий спосіб обчислення що bохcounting розмірність (або розмірності Мінковського) графіка класичної неперервної ніде не диференційовної функції Вейєрштраса

$$
\mathcal{W}(x)=\sum_{n=0}^{+\infty} \lambda^{n} \cos \left(2 \pi N_{b}^{n} x\right),
$$

де $\lambda$ та $N_{b}$ - дійсні числа такі, що $0<\lambda<1, N_{b} \in \mathbb{N}, \lambda N_{b}>1$.

Відмітимо, що попередні роботи, в яких обчислювалась box-counting розмірність графіка функції Вейєрштраса, базувались на таких поняттях теорії динамічних систем як: розмірність Ляпунова еквівалентних притягуючих торів, вкладення графа в атрактор динамічної системи, $b$ відображення пекаря одиничного квадрату, стійкі та нестійкі підмноговиди та ін.

В даній статті ми «обходимо» техніку теорії динамічних систем за допомогою послідовностей графіків $\left(\Gamma_{\mathcal{W}_{m}}\right)_{m \in \mathbb{N}}$, що збігаються до графіка функції Вейєрштраса. Основний інструмент - це ітерована система функцій, тобто сім'я $C^{\infty}$ стискаючих відображень $T_{i}: \mathbb{R}^{2} \rightarrow \mathbb{R}^{2}$, $i=0, \ldots, N_{b}-1$, визначених за формулами

$$
T_{i}(x, y)=\left(\frac{x+i}{N_{b}}, \lambda y+\cos \left(2 \pi \cdot \frac{x+i}{N_{b}}\right)\right),
$$

Нехай $P_{i}$ - нерухома точка $T_{i}$ і $V_{0}=\left\{P_{0}, \ldots, P_{N_{b}-1}\right\}$. Далі для кожного натурального $m$ покладемо $V_{m}=\cup_{i=0}^{N_{b}-1} T_{i}\left(V_{m-1}\right)$. З'єднавши послідовні

2010 Mathematics Subject Classification: 28A80

Keywords: Classical Weierstrass function, box dimension 
точки в множині $V_{m}$ у напрямку зростання абсциси отримаємо граф $\Gamma \mathcal{W}_{m}$. Обчислення розмірності графіка базується на оцінках виду:

$$
C_{\text {inf }}\left|x_{1}-x_{2}\right|^{2-D_{\mathcal{W}}} \leq\left|\mathcal{W}\left(x_{1}\right)-\mathcal{W}\left(x_{2}\right)\right| \leq C_{\text {sup }}\left|x_{1}-x_{2}\right|^{2-D_{\mathcal{W}}}
$$

де $\left[x_{1}, x_{2}\right] \subset[0,1]$ - довільний досить малий інтервалу, $D_{\mathcal{W}}=2+\frac{\ln \lambda}{\ln b}$, а

$C_{\text {inf }}$ i $C_{\text {sup }}$ строго додатні числа. Їх доведення займає лише дві сторінки і не вимагає спеціальних знань, що робить доступним роботу для широкої аудиторії.

\section{INTRODUCTION}

The determination of the box and Hausdorff dimension of the graph of the Weierstrass function has, since long been, a topic of interest. In the following, we show that the box-counting dimension (or Minskowski dimension) can be obtained directly, without using dynamical systems tools.

Let us recall that, given $\lambda \in(0,1)$, and $b$ such that $\lambda b>1+\frac{3 \pi}{2}$, the Weierstrass function

$$
x \in \mathbb{R} \mapsto \mathcal{W}(x)=\sum_{n=0}^{+\infty} \lambda^{n} \cos \left(\pi b^{n} x\right)
$$

is continuous everywhere, while nowhere differentiable. The original proof, by K. Weierstrass [18], can also be found in [17]. It has been completed by the one, now a classical one, in the case where $\lambda b>1$, by G. Hardy [5].

After the works of A. S. Besicovitch and H. D. Ursell [2], it is Benoît Mandelbrot [12] who particularly highlighted the fractal properties of the graph of the Weierstrass function. He also conjectured that the Hausdorff dimension of the graph is $D_{\mathcal{W}}=2+\frac{\ln \lambda}{\ln b}$. Interesting discussions in relation to this question have been given in the book of K. Falconer [4]. A series of results for the box dimension can be found in the works of J. L. Kaplan et al. [8] (where the authors show that it is equal to the Lyapunov dimension of the equivalent attracting torus), in the one of F. Przytycki and M. Urbańki [14], and in those by T. Y. Hu and K-S. Lau [6]. As for the Hausdorff dimension, a proof was given by B. Hunt [7] in 1998 in the case where arbitrary phases are included in each cosinusoidal term of the summation. Recently, K. Barańsky, B. Bárány and J. Romanowska [1] proved that, for any value of the real number $b$, there exists a threshold value $\lambda_{b}$ belonging to the interval $\left(\frac{1}{b}, 1\right)$ such that the aforementioned dimension is equal to $D_{\mathcal{W}}$ for every $b$ in $\left(\lambda_{b}, 1\right)$. Results by W. Shen [16] go further than the ones of [1]. In [9], G. Keller proposes what appears as a much simpler and very original proof.

May one wish to understand the proofs mentioned above, it requires theoretical background in dynamic systems theory. For instance, in the 
work of J. L. Kaplan et al. [8], the authors call for results that cannot be understood without knowledge on the Lyapunov dimension. One may also note that their proof, which enables one to obtain the box-counting dimension of the aforementioned graph, involves sequences revolving around the Gamma Function, Fourier coefficients, integration in the complex plane, definition of a specific measure, the solving of several equations, thus, a lot of technical manipulations (on eleven pages), to yield the result.

Following those results, F. Przytycki and M. Urbańki [14] give a general method leading to the value of this box-counting dimension. It was initially devoted to the calculation of the Hausdorff dimension of the graph. It appears simpler than the one by Kaplan et al., calling for Frostman's lemma [15], [13]. The authors deal with continuous functions $f$ satisfying conditions of the form:

$$
\sup \left\{\left|f\left(a_{1}\right)-f\left(a_{2}\right)\right|: x_{1} \leq a_{1} \leq a_{2} \leq x_{2}\right\} \geq C\left|x_{1}-x_{2}\right|^{\alpha}
$$

for all $\left(x_{1}, x_{2}\right) \in[0,1]^{2}$, where $C$ and $\alpha<1$ denote strictly positive real constants.

In order to apply the results by F. Przytycki and M. Urbańki, one thus requires the estimate $(\star)$, which is not that easy to prove. The same kind of estimate is required to obtain the Hausdorff dimension of the graph. As evoked above, existing works in the literature all call for the theory of dynamical systems.

Until now, the simplest calculation is the one by G. Keller [9], where the author bypasses the Ledrappier-Young theory on hyperbolic measures [10], [11], embedding the graph into an attractor of a dynamical system. The proof requires $b$-baker maps, acting on the unit square. It also requires results on stable and unstable manifolds, as well as results on related fibers.

In our work [3], where we build a Laplacian on the graph of the Weierstrass function $\mathcal{W}$, we came across a simpler means of computing the box dimension of the graph, using a sequence a graphs that approximate the studied one, bypassing all the aforementioned tools. The main computation, which, for any small interval $\left[x_{1}, x_{2}\right] \subset[0,1]$, leads to an estimate of the form:

$$
C_{\text {inf }}\left|x_{1}-x_{2}\right|^{2-D_{\mathcal{W}}} \leq\left|\mathcal{W}\left(x_{1}\right)-\mathcal{W}\left(x_{2}\right)\right| \leq C_{\text {sup }}\left|x_{1}-x_{2}\right|^{2-D_{\mathcal{W}}}
$$

where $D_{\mathcal{W}}=2+\frac{\ln \lambda}{\ln b}$, and $C_{\text {inf }}$ and $C_{\text {sup }}$ denote strictly positive constants, is done in barely two pages, and does not require specific know-ledge, putting the result at the disposal of a wider audience. The key results are exposed in the sequel. 


\section{FRAMEWORK OF THE STUdY}

In this section, we recall results that are developed in [3]. We consider the case when the real number $b$ is an integer, that we thus choose to denote by $N_{b}$.

Notation. We will denote by $\mathbb{N}$ the set of natural integers. In the following, $\lambda$ and $N_{b}$ are two real numbers such that:

$$
0<\lambda<1 \quad N_{b} \in \mathbb{N}, \quad \text { and } \quad \lambda N_{b}>1 .
$$

We will consider the Weierstrass function $\mathcal{W}$, defined, for any $x \in \mathbb{R}$, by:

$$
\mathcal{W}(x)=\sum_{n=0}^{+\infty} \lambda^{n} \cos \left(2 \pi N_{b}^{n} x\right)
$$

Periodic properties of the Weierstrass function. Notice, that for any real number $x$ :

$$
\mathcal{W}(x+1)=\sum_{n=0}^{+\infty} \lambda^{n} \cos \left(2 \pi N_{b}^{n} x+2 \pi N_{b}^{n}\right)=\sum_{n=0}^{+\infty} \lambda^{n} \cos \left(2 \pi N_{b}^{n} x\right)=\mathcal{W}(x)
$$

Hence the study of the Weierstrass function can be restricted to the interval $[0,1)$.

In the sequel, we place ourselves in the Euclidean plane of dimension 2, referred to a direct orthonormal frame. The usual Cartesian coordinates are $(x, y)$.

The restriction $\Gamma_{\mathcal{W}}$ to $[0,1) \times \mathbb{R}$, of the graph of the Weierstrass function, is approximated by means of a sequence of graphs, built through an iterative process. For this purpose, we introduce the iterated function system, i.e. the family of $C^{\infty}$ contractions from $\mathbb{R}^{2}$ to $\mathbb{R}^{2}$ :

$$
\left\{T_{0}, \ldots, T_{N_{b}-1}\right\}
$$

where, for any integer $i$ belonging to $\left\{0, \ldots, N_{b}-1\right\}$, and any $(x, y)$ in $\mathbb{R}^{2}$ :

$$
T_{i}(x, y)=\left(\frac{x+i}{N_{b}}, \lambda y+\cos \left(2 \pi \cdot \frac{x+i}{N_{b}}\right)\right) .
$$

We will denote by:

$$
D_{\mathcal{W}}=2+\frac{\ln \lambda}{\ln N_{b}}
$$

the Hausdorff dimension of $\Gamma_{\mathcal{W}}$ (see [1], [9]).

Proposition 1.1. $\Gamma_{\mathcal{W}}=\bigcup_{i=0}^{N_{b}-1} T_{i}\left(\Gamma_{\mathcal{W}}\right)$. 
Definition 1.2. For any integer $i$ belonging to $\left\{0, \ldots, N_{b}-1\right\}$, let us denote by:

$$
P_{i}=\left(x_{i}, y_{i}\right)=\left(\frac{i}{N_{b}-1}, \frac{1}{1-\lambda} \cos \frac{2 \pi i}{N_{b}-1}\right)
$$

the fixed point of the contraction $T_{i}$.

We will denote by $V_{0}$ the ordered set (according to increasing abscissae), of the points:

$$
\left\{P_{0}, \ldots, P_{N_{b}-1}\right\} \text {. }
$$

The set of points $V_{0}$, where, for any $i$ of $\left\{0, \ldots, N_{b}-2\right\}$, the point $P_{i}$ is linked to the point $P_{i+1}$, constitutes an oriented graph (according to increasing abscissa), that we will denote by $\Gamma_{\mathcal{W}_{0}}$. We will call $V_{0}$ the set of vertices of the graph $\Gamma_{\mathcal{W}_{0}}$.

For any natural integer $m$, we set:

$$
V_{m}=\bigcup_{i=0}^{N_{b}-1} T_{i}\left(V_{m-1}\right) .
$$

The set of points $V_{m}$, where two consecutive points are linked, is an oriented graph (according to increasing abscissa), which we will denote by $\Gamma_{\mathcal{W}_{m}}$. We will call $V_{m}$ the set of vertices of the graph $\Gamma_{\mathcal{W}_{m}}$. We will also denote, in the sequel, by

$$
\mathcal{N}_{m}^{\mathcal{S}}=2 N_{b}^{m}+N_{b}-2
$$

the number of vertices of the graph $\Gamma_{\mathcal{W}_{m}}$, and write:

$$
V_{m}=\left\{P_{0}^{m}, P_{1}^{m}, \ldots, P_{\mathcal{N}_{m}^{\mathcal{S}}-1}^{m}\right\}
$$

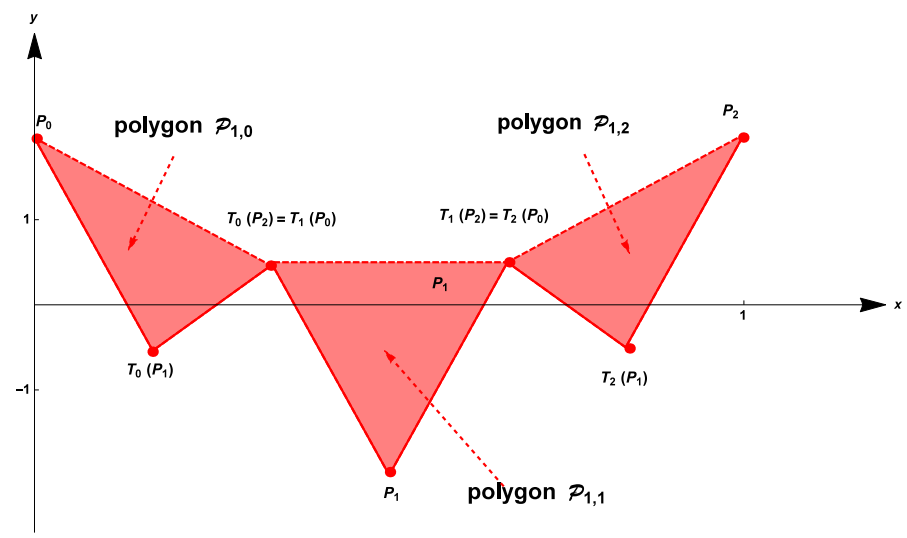

Figure 1.1. The polygons $\mathcal{P}_{1,0}, \mathcal{P}_{1,1}, \mathcal{P}_{1,2}$, in the case where $\lambda=\frac{1}{2}$ and $N_{b}=3$. 


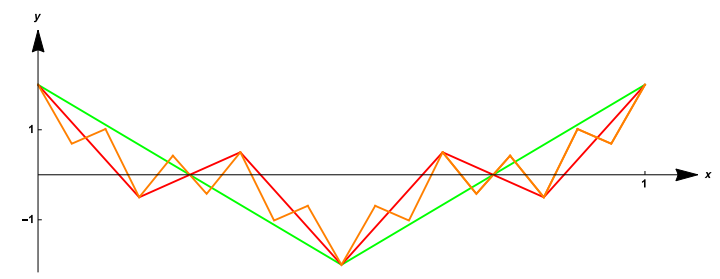

Figure 1.2. The graphs $\Gamma_{\mathcal{W}_{0}}, \Gamma_{\mathcal{W}_{1}}$ (in red), $\Gamma_{\mathcal{W}_{2}}, \Gamma_{\mathcal{W}}$, in the case where $\lambda=\frac{1}{2}$ and $N_{b}=3$.

Definition 1.3 (Consecutive vertices on the graph $\Gamma_{\mathcal{W}}$ ). Two points $X$ and $Y$ of $\Gamma_{\mathcal{W}}$ will be called consecutive vertices of the graph $\Gamma_{\mathcal{W}}$ if there exists a natural integer $m$, and an integer $j$ of $\left\{0, \ldots, N_{b}-2\right\}$, such that:

$$
\left\{\begin{array}{l}
X=\left(T_{i_{1}} \circ \cdots \circ T_{i_{m}}\right)\left(P_{j}\right), \\
Y=\left(T_{i_{1}} \circ \cdots \circ T_{i_{m}}\right)\left(P_{j+1}\right)
\end{array}\right.
$$

or:

$$
\left\{\begin{array}{l}
X=\left(T_{i_{1}} \circ T_{i_{2}} \circ \cdots \circ T_{i_{m}}\right)\left(P_{N_{b}-1}\right), \\
Y=\left(T_{i_{1}+1} \circ T_{i_{2}} \cdots \circ T_{i_{m}}\right)\left(P_{0}\right),
\end{array}\right.
$$

where $\left(i_{1}, \ldots, i_{m}\right) \in\left\{0, \cdots, N_{b}-1\right\}^{m}$.

Definition 1.4. For every $m \in \mathbb{N}$ the $\mathcal{N}_{m}^{\mathcal{S}}$ consecutive vertices of the graph $\Gamma_{\mathcal{W}_{m}}$ are, also, the vertices of $N_{b}^{m}$ simple polygons $\mathcal{P}_{m, j}, 0 \leq j \leq N_{b}^{m}-1$, with $N_{b}$ sides. For any integer $j$ such that $0 \leq j \leq N_{b}^{m}-1$, one obtains each polygon by linking the point number $j$ to the point number $j+1$ if $j=i \bmod N_{b}, 0 \leq i \leq N_{b}-2$, and the point number $j$ to the point number $j-N_{b}+1$ if $j=-1 \bmod N_{b}$. These polygons generate a Borel set of $\mathbb{R}^{2}$.

Definition 1.5 (Word, on the graph $\Gamma_{\mathcal{W}}$ ). Let $m$ be a strictly positive integer. We will call number-letter any integer $\mathcal{M}_{i}$ of $\left\{0, \ldots, N_{b}-1\right\}$, and word of length $|\mathcal{M}|=m$, on the graph $\Gamma_{\mathcal{W}}$, any set of number-letters of the form:

$$
\mathcal{M}=\left(\mathcal{M}_{1}, \ldots, \mathcal{M}_{m}\right)
$$

We will write:

$$
T_{\mathcal{M}}=T_{\mathcal{M}_{1}} \circ \cdots \circ T_{\mathcal{M}_{m}}
$$

Definition 1.6 (Edge relation, on the graph $\Gamma_{\mathcal{W}}$ ). Given a natural integer $m$, two points $X$ and $Y$ of $\Gamma_{\mathcal{W}_{m}}$ will be called adjacent if and only if $X$ and $Y$ are two consecutive vertices of $\Gamma_{\mathcal{W}_{m}}$. We will write:

$$
X \underset{m}{\sim} Y
$$


This edge relation ensures the existence of a word $\mathcal{M}=\left(\mathcal{M}_{1}, \ldots, \mathcal{M}_{m}\right)$ of length $m$, such that $X$ and $Y$ both belong to the iterate:

$$
T_{\mathcal{M}} V_{0}=\left(T_{\mathcal{M}_{1}} \circ \cdots \circ T_{\mathcal{M}_{m}}\right) V_{0}
$$

Given two points $X$ and $Y$ of the graph $\Gamma_{\mathcal{W}}$, we will say that $X$ and $Y$ are adjacent if and only if there exists a natural integer $m$ such that:

$$
X \underset{m}{\sim} Y
$$

Proposition 1.7 (Addresses, on the graph of the Weierstrass function). Given a strictly positive integer $m$, and a word $\mathcal{M}=\left(\mathcal{M}_{1}, \ldots, \mathcal{M}_{m}\right)$ of length $m \in \mathbb{N}^{\star}$, on the graph $\Gamma_{\mathcal{W}_{m}}$, for any integer $j$ of $\left\{1, \ldots, N_{b}-2\right\}$, any $X=T_{\mathcal{M}}\left(P_{j}\right)$ of $V_{m} \backslash V_{0}$, i.e. distinct from one of the $N_{b}$ fixed point $P_{i}$, $0 \leq i \leq N_{b}-1$, has exactly two adjacent vertices, given by:

$$
T_{\mathcal{M}}\left(P_{j+1}\right) \quad \text { and } \quad T_{\mathcal{M}}\left(P_{j-1}\right),
$$

where:

$$
T_{\mathcal{M}}=T_{\mathcal{M}_{1}} \circ \ldots \circ T_{\mathcal{M}_{m}} .
$$

By convention, the adjacent vertices of $T_{\mathcal{M}}\left(P_{0}\right)$ are $T_{\mathcal{M}}\left(P_{1}\right)$ and $T_{\mathcal{M}}\left(P_{N_{b}-1}\right)$, those of $T_{\mathcal{M}}\left(P_{N_{b}-1}\right), T_{\mathcal{M}}\left(P_{N_{b}-2}\right)$ and $T_{\mathcal{M}}\left(P_{0}\right)$.

Notation. For any integer $j$ belonging to $\left\{0, \ldots, N_{b}-1\right\}$, any natural integer $m$, and any word $\mathcal{M}$ of length $m$, we set:

$$
\begin{aligned}
T_{\mathcal{M}}\left(P_{j}\right) & =\left(x\left(T_{\mathcal{M}}\left(P_{j}\right)\right), y\left(T_{\mathcal{M}}\left(P_{j}\right)\right)\right), \\
T_{\mathcal{M}}\left(P_{j+1}\right) & =\left(x\left(T_{\mathcal{M}}\left(P_{j+1}\right)\right), y\left(T_{\mathcal{M}}\left(P_{j+1}\right)\right)\right), \\
L_{m} & =x\left(T_{\mathcal{M}}\left(P_{j+1}\right)\right)-x\left(T_{\mathcal{M}}\left(P_{j}\right)\right)=\frac{1}{\left(N_{b}-1\right) N_{b}^{m}}, \\
h_{j, m} & =y\left(T_{\mathcal{M}}\left(P_{j+1}\right)\right)-y\left(T_{\mathcal{M}}\left(P_{j}\right)\right) .
\end{aligned}
$$

\section{MAin RESUlts}

Theorem 2.1 (An upper bound and a lower bound, for the box-dimension of the graph $\left.\Gamma_{\mathcal{W}}\right)$. For any integer $j$ belonging to $\left\{0,1, \ldots, N_{b}-2\right\}$, each natural integer $m$, and each word $\mathcal{M}$ of length $m$, let us consider the rectangle, whose sides are parallel to the horizontal and vertical axes, of width:

$$
L_{m}=x\left(T_{\mathcal{M}}\left(P_{j+1}\right)\right)-x\left(T_{\mathcal{M}}\left(P_{j}\right)\right)=\frac{1}{\left(N_{b}-1\right) N_{b}^{m}}
$$

and height $\left|h_{j, m}\right|$, such that the points $T_{\mathcal{M}}\left(P_{j}\right)$ and $T_{\mathcal{M}}\left(P_{j+1}\right)$ are two vertices of this rectangle. 
(i) If the integer $N_{b}$ is odd, then:

$$
\begin{aligned}
& L_{m}^{2-} D_{\mathcal{W}}\left(N_{b}-1\right)^{2-D_{\mathcal{W}}} \times \\
& \quad \times\left\{\frac{2}{1-\lambda} \sin \frac{\pi}{N_{b}-1} \min _{0 \leq j \leq N_{b}-1}\left|\sin \frac{\pi(2 j+1)}{N_{b}-1}\right|-\frac{2 \pi}{N_{b}\left(N_{b}-1\right)} \frac{1}{\lambda N_{b}-1}\right\} \leq\left|h_{j, m}\right|
\end{aligned}
$$

(ii) If the integer $N_{b}$ is even, then

$$
\begin{aligned}
L_{m}^{2-D_{\mathcal{W}}\left(N_{b}-\right.} & 1)^{2-D_{\mathcal{W}}} \times \\
& \times \max \left\{\frac{2}{1-\lambda} \sin \frac{\pi}{N_{b}-1} \min _{0 \leq j \leq N_{b}-1}\left|\sin \frac{\pi(2 j+1)}{N_{b}-1}\right|-\right. \\
& \left.\quad-\frac{2 \pi}{N_{b}\left(N_{b}-1\right)} \frac{1}{\lambda N_{b}-1}, \frac{4}{N_{b}^{2}} \frac{1-N_{b}^{-2}}{N_{b}^{2}-1}\right\} \leq\left|h_{j, m}\right|
\end{aligned}
$$

Also:

$$
\left|h_{j, m}\right| \leq \eta_{\mathcal{W}} L_{m}^{2-D_{\mathcal{W}}}\left(N_{b}-1\right)^{2-D_{\mathcal{W}}}
$$

where the real constant $\eta_{\mathcal{W}}$ is given by:

$$
\eta_{\mathcal{W}}=2 \pi^{2}\left\{\frac{\left(2 N_{b}-1\right) \lambda\left(N_{b}^{2}-1\right)}{\left(N_{b}-1\right)^{2}(1-\lambda)\left(\lambda N_{b}^{2}-1\right)}+\frac{2 N_{b}}{\left(\lambda N_{b}^{2}-1\right)\left(\lambda N_{b}^{3}-1\right)}\right\} .
$$

Corollary 2.2. The box-dimension of the graph $\Gamma_{\mathcal{W}}$ is exactly $D_{\mathcal{W}}$.

Proof. By definition of the box-counting dimension $D_{\mathcal{W}}$ (we refer, for instance, to [4]), the smallest number of squares, the side length of which is at most equal to $L_{m}$, that can cover the graph $\Gamma_{\mathcal{W}}$ on $[0,1)$, obeys, approximately, a power law of the form:

$$
c L_{m}^{-D_{\mathcal{W}}}, \quad c>0 \text {. }
$$

Let us set

$$
\begin{array}{r}
C=\max \left\{\left\{\frac{2}{1-\lambda} \sin \frac{\pi}{N_{b}-1} \min _{0 \leq j \leq N_{b}-1}\left|\sin \frac{\pi(2 j+1)}{N_{b}-1}\right|-\frac{2 \pi}{N_{b}\left(N_{b}-1\right)} \frac{1}{\lambda N_{b}-1}\right\},\right. \\
\left.\frac{4}{N_{b}^{2}} \frac{1-N_{b}^{-2}}{N_{b}^{2}-1}, \eta_{\mathcal{W}}\right\}
\end{array}
$$

and consider the subdivision of the interval $[0,1)$ into:

$$
N_{m}=\frac{1}{L_{m}}=\left(N_{b}-1\right) N_{b}^{m}
$$

sub-intervals of length $L_{m}$. One has to determine a natural integer $\tilde{N}_{m}$ such that the graph of $\Gamma_{\mathcal{W}}$ on $[0,1)$ can be covered by $N_{m} \times \tilde{N}_{m}$ squares of side $L_{m}$. By considering, the vertical amplitude of the graph, one gets:

$$
\tilde{N}_{m}=\left\lfloor\frac{C L_{m}^{2-D_{\mathcal{W}}}}{L_{m}}\right\rfloor+1, \quad \text { i.e. } \quad \tilde{N}_{m}=\left\lfloor C L_{m}^{\left.1-D_{\mathcal{W}}\right\rfloor+1 .}\right.
$$


Box counting dimension of the graph of the Weierstrass function

Thus,

$$
N_{m} \times \tilde{N}_{m}=\frac{\tilde{N}_{m}}{L_{m}}=\frac{1}{L_{m}}\left\lfloor C L_{m}^{1-D \mathcal{W}}\right\rfloor+\frac{1}{L_{m}} .
$$

The integer $N_{m} \times \tilde{N}_{m}$ then obeys a power law of the form

$$
N_{m} \times \tilde{N}_{m} \approx c L_{m}^{-D_{\mathcal{W}}}
$$

where $c$ denotes a strictly positive constant.

Proof of Theorem 2.1. Preliminary computations. For any pair of integers $\left(i_{m}, j\right)$ of $\left\{0, \ldots, N_{b}-\overline{2\}^{2}}\right.$

$$
T_{i_{m}}\left(P_{j}\right)=\left(\frac{x_{j}+i_{m}}{N_{b}}, \lambda y_{j}+\cos \left(2 \pi \frac{x_{j}+i_{m}}{N_{b}}\right)\right) .
$$

For any triple of integers $\left(i_{m}, i_{m-1}, j\right)$ of $\left\{0, \ldots, N_{b}-2\right\}^{3}$

$$
\begin{aligned}
& T_{i_{m-1}}\left(T_{i_{m}}\left(P_{j}\right)\right)= \\
& =\left(\frac{\frac{x_{j}+i_{m}}{N_{b}}+i_{m-1}}{N_{b}}, \lambda^{2} y_{j}+\lambda \cos \left(2 \pi \frac{x_{j}+i_{m}}{N_{b}}\right)+\cos \left(2 \pi \frac{\frac{x_{j}+i_{m}}{N_{b}}+i_{m-1}}{N_{b}}\right)\right) \\
& =\left(\frac{x_{j}+i_{m}}{N_{b}^{2}}+\frac{i_{m-1}}{N_{b}}, \lambda^{2} y_{j}+\lambda \cos \left(2 \pi \frac{x_{j}+i_{m}}{N_{b}}\right)+\cos \left(2 \pi\left(\frac{x_{j}+i_{m}}{N_{b}^{2}}+\frac{i_{m-1}}{N_{b}}\right)\right)\right) .
\end{aligned}
$$

For any quadruple of integers $\left(i_{m}, i_{m-1}, i_{m-2}, j\right)$ of $\left\{0, \ldots, N_{b}-2\right\}^{4}$

$$
\begin{gathered}
T_{i_{m-2}}\left(T_{i_{m-1}}\left(T_{i_{m}}\left(P_{j}\right)\right)\right)=\left(\frac{x_{j}+i_{m}}{N_{b}^{3}}+\frac{i_{m-1}}{N_{b}^{2}}+\frac{i_{m-2}}{N_{b}}, \lambda^{3} y_{j}+\lambda^{2} \cos \left(2 \pi \frac{x_{j}+i_{m}}{N_{b}}\right)+\right. \\
\left.+\lambda \cos \left(2 \pi\left(\frac{x_{j}+i_{m}}{N_{b}^{2}}+\frac{i_{m-1}}{N_{b}}\right)\right)+\cos \left(2 \pi\left(\frac{x_{j}+i_{m}}{N_{b}^{3}}+\frac{i_{m-1}}{N_{b}^{2}}+\frac{i_{m-2}}{N_{b}}\right)\right)\right) .
\end{gathered}
$$

Given a strictly positive integer $m$, and two points $X$ and $Y$ of $V_{m}$ such that:

$$
X \underset{m}{\sim} Y
$$

there exists a word $\mathcal{M}$ of length $|\mathcal{M}|=m$, on the graph $\Gamma_{\mathcal{W}}$, and an integer $j$ of $\left\{0, \ldots, N_{b}-2\right\}^{2}$, such that:

$$
X=T_{\mathcal{M}}\left(P_{j}\right), \quad Y=T_{\mathcal{M}}\left(P_{j+1}\right) .
$$

Let us write $T_{\mathcal{M}}$ under the form:

$$
T_{\mathcal{M}}=T_{i_{m}} \circ T_{i_{m-1}} \circ \ldots \circ T_{i_{1}},
$$

where $\left(i_{1}, \ldots, i_{m}\right) \in\left\{0, \ldots, N_{b}-1\right\}^{m}$.

One has then:

$$
x\left(T_{\mathcal{M}}\left(P_{j}\right)\right)=\frac{x_{j}}{N_{b}^{m}}+\sum_{k=1}^{m} \frac{i_{k}}{N_{b}^{k}}, \quad x\left(T_{\mathcal{M}}\left(P_{j+1}\right)\right)=\frac{x_{j+1}}{N_{b}^{m}}+\sum_{k=1}^{m} \frac{i_{k}}{N_{b}^{k}},
$$


and:

$$
\left\{\begin{array}{l}
y\left(T_{\mathcal{M}}\left(P_{j}\right)\right)=\lambda^{m} y_{j}+\sum_{k=1}^{m} \lambda^{m-k} \cos \left(2 \pi\left(\frac{x_{j}}{N_{b}^{k}}+\sum_{\ell=0}^{k} \frac{i_{m-\ell}}{N_{b}^{k-\ell}}\right)\right) \\
y\left(T_{\mathcal{M}}\left(P_{j+1}\right)\right)=\lambda^{m} y_{j+1}+\sum_{k=1}^{m} \lambda^{m-k} \cos \left(2 \pi\left(\frac{x_{j+1}}{N_{b}^{k}}+\sum_{\ell=0}^{k} \frac{i_{m-\ell}}{N_{b}^{k-\ell}}\right)\right)
\end{array}\right.
$$

Determination of a lower bound. Let us note that:

$$
\begin{aligned}
h_{j, m} & -\lambda^{m}\left(y_{j+1}-y_{j}\right)= \\
& =\sum_{k=1}^{m} \lambda^{m-k}\left\{\cos \left(2 \pi\left(\frac{x_{j+1}}{N_{b}^{k}}+\sum_{\ell=0}^{k} \frac{i_{m-\ell}}{N_{b}^{k-\ell}}\right)\right)-\cos \left(2 \pi\left(\frac{x_{j}}{N_{b}^{k}}-\sum_{\ell=0}^{k} \frac{i_{m-\ell}}{N_{b}^{k-\ell}}\right)\right)\right\} \\
& =-2 \sum_{k=1}^{m} \lambda^{m-k} \sin \left(\pi \frac{x_{j+1}-x_{j}}{N_{b}^{k}}\right) \sin \left(2 \pi\left(\frac{x_{j+1}+x_{j}}{2 N_{b}^{k}}+\sum_{\ell=0}^{k} \frac{i_{m-\ell}}{N_{b}^{k-\ell}}\right)\right) .
\end{aligned}
$$

Taking into account:

$$
\begin{aligned}
\lambda^{m}\left(y_{j+1}-y_{j}\right) & =\frac{\lambda^{m}}{1-\lambda}\left(\cos \frac{2 \pi(j+1)}{N_{b}-1}-\cos \frac{2 \pi j}{N_{b}-1}\right) \\
& =\frac{-2 \lambda^{m}}{1-\lambda} \sin \frac{2 \pi(j+1-j)}{2\left(N_{b}-1\right)} \sin \frac{2 \pi(j+1+j)}{2\left(N_{b}-1\right)} \\
& =\frac{-2 \lambda^{m}}{1-\lambda} \sin \frac{\pi}{N_{b}-1} \sin \frac{\pi(2 j+1)}{N_{b}-1},
\end{aligned}
$$

the triangular inequality leads then to:

$$
\begin{aligned}
& \left|y\left(T_{\mathcal{M}}\left(P_{j+1}\right)\right)-y\left(T_{\mathcal{M}}\left(P_{j}\right)\right)\right|= \\
& =\mid \lambda^{m}\left(y_{j+1}-y_{j}\right)-2 \sum_{k=1}^{m} \lambda^{m-k} \sin \frac{\pi}{N_{b}^{k+1}\left(N_{b}-1\right)} \sin \left(\frac{\pi(2 j+1)}{N_{b}^{k+1}\left(N_{b}-1\right)}+2 \pi \sum_{\ell=0}^{k} \frac{i_{m-\ell}}{\left.N_{b}^{k-\ell}\right)} \mid\right. \\
& \geq\left|\lambda^{m}\right| y_{j+1}-y_{j}\left|-2 \sum_{k=1}^{m} \lambda^{m-k}\right| \sin \frac{\pi}{N_{b}^{k+1}\left(N_{b}-1\right)} \sin \left(\frac{\pi(2 j+1)}{N_{b}^{k+1}\left(N_{b}-1\right)}+2 \pi \sum_{\ell=0}^{k} \frac{i_{m}-\ell}{\left.N_{b}^{k-\ell}\right)}\right) \mid \\
& =\lambda^{m}\left|\frac{2}{1-\lambda} \sin \frac{\pi}{N_{b}-1} \cdot\right| \sin \frac{\pi(2 j+1)}{N_{b}-1} \mid- \\
& \quad-2 \sum_{k=1}^{m} \lambda^{-k} \sin \frac{\pi}{N_{b}^{k+1}\left(N_{b}-1\right)} \cdot\left|\sin \left(\frac{\pi(2 j+1)}{N_{b}^{k+1}\left(N_{b}-1\right)}+2 \pi \sum_{\ell=0}^{k} \frac{i_{m-\ell}}{\left.N_{b}^{k-\ell}\right)}\right)\right| .
\end{aligned}
$$


One also has:

$$
\begin{aligned}
2 \sum_{k=1}^{m} \lambda^{-k} \sin \frac{\pi}{N_{b}^{k+1}\left(N_{b}-1\right)}\left|\sin \left(\frac{\pi(2 j+1)}{N_{b}^{k+1}\left(N_{b}-1\right)}+2 \pi \sum_{\ell=0}^{k} \frac{i_{m-\ell}}{N_{b}^{k-\ell}}\right)\right| \leq \\
\leq 2 \sum_{k=1}^{m} \lambda^{-k} \sin \frac{\pi}{N_{b}^{k+1}\left(N_{b}-1\right)} \leq 2 \sum_{k=1}^{m} \lambda^{-k} \frac{\pi}{N_{b}^{k+1}\left(N_{b}-1\right)} \\
=\frac{2 \pi}{N_{b}\left(N_{b}-1\right)} \sum_{k=1}^{m} \frac{1}{\lambda^{k} N_{b}^{k}}=\frac{2 \pi}{N_{b}\left(N_{b}-1\right)} \lambda^{-1} N_{b}^{-1} \frac{1-\lambda^{-m} N_{b}^{-m}}{1-\lambda^{-1} N_{b}^{-1}} \\
\leq \frac{2 \pi}{N_{b}\left(N_{b}-1\right)} \lambda^{-1} N_{b}^{-1} \frac{1}{1-\lambda^{-1} N_{b}^{-1}}=\frac{2 \pi}{N_{b}\left(N_{b}-1\right)} \frac{1}{\lambda N_{b}-1}
\end{aligned}
$$

which yields:

$$
\begin{aligned}
-2 \sum_{k=1}^{m} \lambda^{-k} \sin \frac{\pi}{N_{b}^{k+1}\left(N_{b}-1\right)} \cdot \mid \sin \left(\frac{\pi(2 j+1)}{N_{b}^{k+1}\left(N_{b}-1\right)}+2 \pi \sum_{\ell=0}^{k} \frac{i_{m-\ell}}{\left.N_{b}^{k-\ell}\right) \mid \geq}\right. & \\
& \geq-\frac{2 \pi}{N_{b}\left(N_{b}-1\right)} \frac{1}{\lambda N_{b}-1} .
\end{aligned}
$$

Thus:

$$
\begin{aligned}
& \frac{2}{1-\lambda} \sin \frac{\pi}{N_{b}-1} \cdot\left|\sin \frac{\pi(2 j+1)}{N_{b}-1}\right|- \\
& -2 \sum_{k=1}^{m} \lambda^{-k} \sin \frac{\pi}{N_{b}^{k+1}\left(N_{b}-1\right)} \cdot \mid \sin \left(\frac{\pi(2 j+1)}{N_{b}^{k+1}\left(N_{b}-1\right)}+2 \pi \sum_{\ell=0}^{k} \frac{i_{m-\ell}}{\left.N_{b}^{k-\ell}\right)} \mid \geq\right. \\
& \quad \geq \frac{2}{1-\lambda} \sin \frac{\pi}{N_{b}-1} \cdot\left|\sin \frac{\pi(2 j+1)}{N_{b}-1}\right|-\frac{2 \pi}{N_{b}\left(N_{b}-1\right)} \frac{1}{\lambda N_{b}-1} .
\end{aligned}
$$

Lemma 2.2.1. The following result holds: for $0 \leq j \leq N_{b}-1$ :

$$
\sin \frac{\pi(2 j+1)}{N_{b}-1}=0 \text { if and only if } N_{b} \text { is even and } j=\frac{N_{b}}{2}-1 \text {. }
$$

Proof. Since $0 \leq j \leq N_{b}-1$, one has:

$$
1 \leq 2 j+1 \leq 2 N_{b}-1 \text { and thus } 0<\frac{2 j+1}{N_{b}-1} \leq 2+\frac{1}{N_{b}-1} .
$$

Then, $\sin \frac{\pi(2 j+1)}{N_{b}-1}=0$ if and only if

$$
\frac{2 j+1}{N_{b}-1}=1 \quad \text { or } \quad \frac{2 j+1}{N_{b}-1}=2 .
$$

The second case has to be rejected, since it would lead to

$$
j=N_{b}-\frac{3}{2} \notin \mathbb{N}
$$


The only possibility is thus when $N_{b}$ is an even number:

$$
j=\frac{N_{b}}{2}-1 \text {. }
$$

The converse is obvious.

First case: $\sin \frac{\pi(2 j+1)}{N_{b}-1} \neq 0$. One has then:

$$
\left|\sin \frac{\pi(2 j+1)}{N_{b}-1}\right| \geq \min _{0 \leq j \leq N_{b}-1}\left|\sin \frac{\pi(2 j+1)}{N_{b}-1}\right|=\left|\sin \frac{\pi}{N_{b}-1}\right| \geq \frac{2}{N_{b}-1} .
$$

This leads to:

$$
\begin{aligned}
\frac{2}{1-\lambda} \sin \frac{\pi}{N_{b}-1} \cdot\left|\sin \frac{\pi(2 j+1)}{N_{b}-1}\right|- \\
-2 \sum_{k=1}^{m} \lambda^{-k} \sin \frac{\pi}{N_{b}^{k+1}\left(N_{b}-1\right)} \cdot \mid \sin \left(\frac{\pi(2 j+1)}{N_{b}^{k+1}\left(N_{b}-1\right)}+2 \pi \sum_{\ell=0}^{k} \frac{i_{m-\ell}}{\left.N_{b}^{k-\ell}\right)} \mid \geq\right. \\
\geq \frac{2}{1-\lambda} \sin \frac{\pi}{N_{b}-1} \sin \frac{\pi}{N_{b}-1}-\frac{2 \pi}{N_{b}\left(N_{b}-1\right)} \frac{1}{\lambda N_{b}-1} \\
\geq \frac{2}{1-\lambda} \frac{4}{\left(N_{b}-1\right)^{2}}-\frac{2 \pi}{N_{b}\left(N_{b}-1\right)} \frac{1}{\lambda N_{b}-1} \\
=\frac{2}{N_{b}-1}\left\{\frac{4}{1-\lambda} \frac{1}{N_{b}-1}-\frac{\pi}{N_{b}} \frac{1}{\lambda N_{b}-1}\right\} \\
=\frac{2\left(4 N_{b}\left(\lambda N_{b}-1\right)-\pi(1-\lambda)\left(N_{b}-1\right)\right)}{N_{b}\left(N_{b}-1\right)(1-\lambda)\left(\lambda N_{b}-1\right)} .
\end{aligned}
$$

The function

$$
\lambda \longmapsto 4 N_{b}\left(\lambda N_{b}-1\right)-\pi(1-\lambda)\left(N_{b}-1\right)
$$

is affine and strictly increasing in $\lambda$, and quadratic and strictly increasing in $N_{b}$, for strictly positive values of $N_{b}$. This ensures the positivity of:

$$
\begin{aligned}
& \frac{2}{1-\lambda} \sin \frac{\pi}{N_{b}-1} \cdot\left|\sin \frac{\pi(2 j+1)}{N_{b}-1}\right|- \\
& \quad-2 \sum_{k=1}^{m} \lambda^{-k} \sin \frac{\pi}{N_{b}^{k+1}\left(N_{b}-1\right)} \cdot\left|\sin \left(\frac{\pi(2 j+1)}{N_{b}^{k+1}\left(N_{b}-1\right)}+2 \pi \sum_{\ell=0}^{k} \frac{i_{m-\ell}}{N_{b}^{k-\ell}}\right)\right| .
\end{aligned}
$$

Second case: $\sin \frac{\pi(2 j+1)}{N_{b}-1}=0$. One has then:

$$
\begin{aligned}
\mid y\left(T_{\mathcal{M}}\left(P_{j+1}\right)\right)-y & \left(T_{\mathcal{M}}\left(P_{j}\right)\right) \mid \geq \\
& \geq 2 \lambda^{m}\left|\sum_{k=1}^{m} \lambda^{-k} \sin \frac{\pi}{N_{b}^{k+1}} \cdot\right| \sin \left(\frac{\pi}{N_{b}^{k+1}}+2 \pi \sum_{\ell=0}^{k} \frac{i_{m-\ell}}{N_{b}^{k-\ell}}\right)|| .
\end{aligned}
$$


Box counting dimension of the graph of the Weierstrass function

Thanks to the periodic properties of the sine function, one may only consider the case when:

$$
0 \leq \frac{\pi}{N_{b}^{k+1}}+2 \pi \sum_{\ell=0}^{k} \frac{i_{m-\ell}}{N_{b}^{k-\ell}} \leq \frac{\pi}{2} .
$$

Thus,

$$
\begin{aligned}
\mid y\left(T_{\mathcal{M}}\left(P_{j+1}\right)\right)- & y\left(T_{\mathcal{M}}\left(P_{j}\right)\right) \mid \geq \sum_{k=1}^{m} \lambda^{-k} \frac{2}{N_{b}^{k+1}}\left\{\frac{2}{N_{b}^{k+1}}+2 \sum_{\ell=0}^{k} \frac{i_{m-\ell}}{N_{b}^{k-\ell}}\right\} \\
& \geq \sum_{k=1}^{m} \lambda^{-k} \frac{2}{N_{b}^{k+1}}\left\{\frac{2}{N_{b}^{k+1}}\right\} \\
& =\frac{4 \lambda^{-1}}{N_{b}^{4}} \frac{1-\lambda^{-m} N_{b}^{-2 m}}{1-\lambda^{-1} N_{b}^{-2}} \\
& =\frac{4}{N_{b}^{2}\left(N_{b}-1\right)^{2}} \frac{1-\lambda^{-m} N_{b}^{-m}}{\lambda N_{b}-1}=\frac{4}{N_{b}^{2}} \frac{1-N_{b}^{-2}}{N_{b}^{2}-1} .
\end{aligned}
$$

General case. The above results enable us to obtain the predominant term of the lower bound of $\left|y\left(T_{\mathcal{M}}\left(P_{j+1}\right)\right)-y\left(T_{\mathcal{M}}\left(P_{j}\right)\right)\right|$, which is thus:

$$
\lambda^{m}=e^{m\left(D_{\mathcal{W}}-2\right) \ln N_{b}}=N_{b}^{m\left(D_{\mathcal{W}}-2\right)}=L_{m}^{2-D_{\mathcal{W}}}\left(N_{b}-1\right)^{2-D_{\mathcal{W}}} .
$$

Determination of an upper bound. One has

$$
\begin{aligned}
& \left|h_{j, m}\right| \leq \frac{2 \lambda^{m}}{1-\lambda} \frac{\pi^{2}(2 j+1)}{\left(N_{b}-1\right)^{2}}+2 \sum_{k=1}^{m} \lambda^{m-k} \pi\left\{\frac{2 j+1}{\left(N_{b}-1\right) N_{b}^{k}}+2 \sum_{\ell=0}^{k} \frac{i_{m-\ell}}{N_{b}^{k-\ell}}\right\} \frac{\pi}{\left(N_{b}-1\right) N_{b}^{k}} \\
& =\frac{2 \lambda^{m}}{1-\lambda} \frac{\pi^{2}(2 j+1)}{\left(N_{b}-1\right)^{2}}+\frac{2 \pi^{2} \lambda^{m}}{N_{b}-1} \sum_{k=1}^{m}\left\{\frac{(2 j+1) \lambda^{-k}}{\left(N_{b}-1\right) N_{b}^{2 k}}+2 \sum_{\ell=0}^{k} \frac{i_{m-\ell} \lambda^{-k}}{N_{b}^{2 k-\ell}}\right\} \\
& =\frac{2 \lambda^{m}}{1-\lambda} \frac{\pi^{2}(2 j+1)}{\left(N_{b}-1\right)^{2}}+ \\
& +\frac{2 \pi^{2} \lambda^{m}}{N_{b}-1}\left\{\frac{\lambda^{-1} N_{b}^{-2}(2 j+1)}{\left(N_{b}-1\right)} \frac{\left(1-\lambda^{-m} N_{b}^{-2 m}\right)}{1-\lambda^{-1} N_{b}^{-2}}+2 \sum_{k=1}^{m} \frac{\left(N_{b}-1\right) \lambda^{-k}}{N_{b}^{2 k}} \frac{1-N_{b}^{-k-1}}{1-N_{b}^{-1}}\right\} \\
& \leq \frac{2 \lambda^{m}}{1-\lambda} \frac{\pi^{2}\left(2 N_{b}-1\right)}{\left(N_{b}-1\right)^{2}}+\frac{2 \pi^{2} \lambda^{m}}{N_{b}-1} \frac{\left(2 N_{b}-1\right)}{\left(N_{b}-1\right)} \frac{\left(1-\lambda^{-m} N_{b}^{-2 m}\right)}{\lambda N_{b}^{2}-1} \\
& +\frac{2 \pi^{2} \lambda^{m}}{N_{b}-1} 2 \frac{\lambda^{-1} N_{b}^{-2}\left(N_{b}-1\right)\left(1-\lambda^{-m} N_{b}^{-2 m}\right)}{\left(1-N_{b}^{-1}\right)\left(1-\lambda^{-1} N_{b}^{-2}\right)}
\end{aligned}
$$




$$
\begin{aligned}
& -\frac{2 \pi^{2} \lambda^{m}}{N_{b}-1} 2 \frac{\lambda^{-1} N_{b}^{-3}\left(N_{b}-1\right)\left(1-\lambda^{-m} N_{b}^{-3 m}\right)}{\left(1-N_{b}^{-1}\right)\left(1-\lambda^{-1} N_{b}^{-3}\right)} \\
& \leq \frac{2 \lambda^{m}}{1-\lambda} \frac{\pi^{2}\left(2 N_{b}-1\right)}{\left(N_{b}-1\right)^{2}}+\frac{2 \pi^{2} \lambda^{m}}{N_{b}-1} \frac{\left(2 N_{b}-1\right)}{\left(N_{b}-1\right)} \frac{1}{\lambda N_{b}^{2}-1} \\
& +\frac{4 \pi^{2} N_{b} \lambda^{m}}{N_{b}-1}\left\{\frac{1}{\lambda N_{b}^{2}-1}-\frac{1}{\lambda N_{b}^{3}-1}\right\} \\
& =2 \pi^{2} \lambda^{m}\left\{\frac{\left(2 N_{b}-1\right) \lambda\left(N_{b}^{2}-1\right)}{\left(N_{b}-1\right)^{2}(1-\lambda)\left(\lambda N_{b}^{2}-1\right)}+\frac{2 N_{b}}{\left(\lambda N_{b}^{2}-1\right)\left(\lambda N_{b}^{3}-1\right)}\right\} .
\end{aligned}
$$

Since

$$
x\left(T_{\mathcal{M}}\left(P_{j+1}\right)\right)-x\left(T_{\mathcal{M}}\left(P_{j}\right)\right)=\frac{1}{\left(N_{b}-1\right) N_{b}^{m}}
$$

and

$$
D_{\mathcal{W}}=2+\frac{\ln \lambda}{\ln N_{b}}, \quad \lambda=e^{\left(D_{\mathcal{W}}-2\right) \ln N_{b}}=N_{b}^{\left(D_{\mathcal{W}}-2\right)},
$$

one has thus

$$
\begin{aligned}
\left|h_{j, m}\right| \leq 2 \pi^{2} & L_{m}^{2-} D_{\mathcal{W}}\left(N_{b}-1\right)^{2-D_{\mathcal{W}} \times} \\
& \times\left\{\frac{\left(2 N_{b}-1\right) \lambda\left(N_{b}^{2}-1\right)}{\left(N_{b}-1\right)^{2}(1-\lambda)\left(\lambda N_{b}^{2}-1\right)}+\frac{2 N_{b}}{\left(\lambda N_{b}^{2}-1\right)\left(\lambda N_{b}^{3}-1\right)}\right\} .
\end{aligned}
$$

Theorem 2.1 is completed.

Remark 2.3. In [7] B. Hunt uses the fact that the Hausdorff dimension of a fractal set $\mathcal{F}$ can be obtained by means of what is called the $t$-energy, $t \in \mathbb{R}$, of a Borel measure supported on $\mathcal{F}$ (one may refer to [4], for instance):

$$
I_{t}(\mu)=\iint \frac{d \mu(x) d \mu\left(x^{\prime}\right)}{\left|x-x^{\prime}\right|^{t}}
$$

which enables one to obtain:

$$
\operatorname{dim} \mathcal{F}=\sup \left\{t \in \mathbb{R}, \mid \mu \text { supported on } \mathcal{F}, I_{t}(\mu)<+\infty\right\} .
$$

A lower bound $t_{0}$ of the Hausdorff dimension can thus be obtained by building a measure $\mu$ supported on $\mathcal{F}$ such that:

$$
I_{t_{0}}(\mu)<+\infty \text {. }
$$

B. Hunt proceeds as follows: he introduces the measure $\mu_{\mathcal{W}}$ supported on $\Gamma_{\mathcal{W}}$, induced by the Lebesgue measure $\mu$ on $[0,1]$. Thus

$$
I_{t}\left(\mu_{\mathcal{W}}\right)=\iint \frac{d \mu_{\mathcal{W}}(x) d \mu_{\mathcal{W}}\left(x^{\prime}\right)}{\left\{\left|x-x^{\prime}\right|^{2}+\left|\mathcal{W}(x)-\mathcal{W}\left(x^{\prime}\right)\right|^{2}\right\}^{\frac{t}{2}}}
$$


We could also have used a similar argument since, in our case:

$$
\left|x-x^{\prime}\right|^{2-D_{\mathcal{W}}} \lesssim\left|\mathcal{W}(x)-\mathcal{W}\left(x^{\prime}\right)\right| \lesssim\left|x-x^{\prime}\right|^{2-D_{\mathcal{W}}}
$$

Acknowledgements. The author would like to thank the anonymous referee for his careful reading, and his very pertinent suggestions and advices, which helped a lot improving the original work.

\section{REFERENCES}

[1] Krzysztof Barański, Balázs Bárány, Julia Romanowska. On the dimension of the graph of the classical Weierstrass function. Adv. Math., 265:32-59, 2014.

[2] A. S. Besicovitch, H. D. Ursell. Sets of fractional dimensions (V): on dimensional numbers of some continuous curves. J. London Math. Soc., s1-12(1):18-25, 1937.

[3] Claire David. Laplacian, on the graph of the Weierstrass function. arXiv:1703.03371.

[4] K. J. Falconer. The geometry of fractal sets, volume 85 of Cambridge Tracts in Mathematics. Cambridge University Press, Cambridge, 1986.

[5] G. H. Hardy. Theorems Connected with Maclaurin's Test for the Convergence of Series. Proc. London Math. Soc. (2), 9:126-144, 1911.

[6] Tian You Hu, Ka-Sing Lau. Fractal dimensions and singularities of the Weierstrass type functions. Trans. Amer. Math. Soc., 335(2):649-665, 1993.

[7] Brian R. Hunt. The Hausdorff dimension of graphs of Weierstrass functions. Proc. Amer. Math. Soc., 126(3):791-800, 1998.

[8] James L. Kaplan, John Mallet-Paret, James A. Yorke. The Lyapunov dimension of a nowhere differentiable attracting torus. Ergodic Theory Dynam. Systems, 4(2):261-281, 1984.

[9] Gerhard Keller. A simpler proof for the dimension of the graph of the classical Weierstrass function. Ann. Inst. Henri Poincaré Probab. Stat., 53(1):169-181, 2017.

[10] F. Ledrappier, L.-S. Young. The metric entropy of diffeomorphisms. II. Relations between entropy, exponents and dimension. Ann. of Math. (2), 122(3):540-574, 1985.

[11] François Ledrappier. On the dimension of some graphs. In Symbolic dynamics and its applications (New Haven, CT, 1991), volume 135 of Contemp. Math., 285-293. Amer. Math. Soc., Providence, RI, 1992.

[12] Benoit B. Mandelbrot. Fractals: form, chance, and dimension. W. H. Freeman and Co., San Francisco, Calif., revised edition, 1977. Translated from the French.

[13] Yakov B. Pesin. Dimension theory in dynamical systems. Chicago Lectures in Mathematics. University of Chicago Press, Chicago, IL, 1997. Contemporary views and applications.

[14] F. Przytycki, M. Urbański. On the Hausdorff dimension of some fractal sets. Studia Math., 93(2):155-186, 1989.

[15] Feliks Przytycki, Mariusz Urbański. Conformal fractals: ergodic theory methods, volume 371 of London Mathematical Society Lecture Note Series. Cambridge University Press, Cambridge, 2010.

[16] Weixiao Shen. Hausdorff dimension of the graphs of the classical Weierstrass functions. Math. Z., 289(1-2):223-266, 2018.

[17] E. C. Titchmarsh. The theory of functions. Oxford University Press, Oxford, second edition, 1939. 
[18] K. Weierstrass. Über kontinuierliche Funktionen eines reellen arguments, die für keinen Wert des letzteren einen bestimmten Differentialquotienten besitzen. Journal für die reine und angewandte Mathematik, 79:29-31, 1875.

Received: December 5, 2017, accepted: July 1, 2018.

Claire David

Sorbonne Université, CNRS, Laboratoire Jacques-Louis Lions, 4, place Jussieu 75005, Paris, France

Email: Claire.David@upmc.fr

ORCID: orcid.org/0000-0002-4729-0733 